Journal of Animal and Veterinary Advances 11 (15): 2611-2617, 2012

ISSN: $1680-5593$

(C) Medwell Journals, 2012

\title{
Angiogenic and Osteogenic Responses Related to Femoral Neck Fractures Treated at Different Fracture-To-Surgery Intervals in a Canine Model
}

\author{
Y.S. Gao, H. Ding, X.W. Yu and C.Q. Zhang \\ Department of Orthopedic Surgery, Shanghai Sixth People's Hospital, \\ Jiao Tong University, 200233 Shanghai, China
}

\begin{abstract}
The aim of current prospective study was to investigate systemic changes in angiogenic factor VEGF and osteogenic factor IGF-1 and the local expression of BMP-2 and Runx-2 in femoral neck fractures of canines that were treated at different fracture-to-surgery intervals. About 24 dogs had surgically induced femoral neck fractures which were divided randomly and equally into three groups. In group A, the fractures were reduced and stabilized by paralleled pins immediately while they were managed 3 days later in group B and 3 weeks later in group C. Pre and post-operative peripheral serum was prepared for detection of systemic concentrations of VEGF and IGF-1 via ELISA Method. Postoperative radiological and histological examinations were employed to observe fracture union and occurrence of Osteonecrosis of the Femoral Head (ONFH). Local expressions of BMP-2 and Runx-2 were detected via immunohistochemical staining. Another 2 dogs had fractures untreated for negative control. Systemic changes in VEGF and IGF-1 were shown to share similarities when fractures were treated at different fracture-to-surgery intervals or left untreated. Moreover, the local expression of BMP-2 and Runx-2 were not statistically different in all treated and untreated animals. The manner of the response of systemic expression of VEGF and IGF-1 and local expression of BMP-2 and Runx-2 showed great similarity when femoral neck fractures were treated at different fracture-to-surgery intervals. Angiogenic and osteogenic responses might not be responsible for fracture-induced $\mathrm{ONFH}$.
\end{abstract}

$\underline{\text { Key words: Femoral neck fractures, angiogenesis, osteogenesis, Canine Model, dogs, China }}$

\section{INTRODUCTION}

Traumatic Osteonecrosis of the Femoral Head $(\mathrm{ONFH})$ is a severe sequela of femoral neck fractures. Although, the pathogenesis of fracture-induced $\mathrm{ONFH}$ is unclear, it is thought that primary trauma and the quality of reduction and internal fixation are principal determinants (Lu-Yao et al., 2004; Kyle, 2009). However, it is still controversial whether the reduction of the fracture-to-surgery interval could prevent secondary $\mathrm{ONFH}$ and its underlying association with osteogenic and angiogenic responses are unclear (Upadhyay et al., 2004). In terms of pathophysiology, regional osteogenesis and angiogenesis are decreased and the repair ability is impaired which ultimately contribute to an imbalance of bone remodeling. The natural history of the disease in the majority of patients is a progression to degeneration of the hip joint (Jones and Hungerford, 2007). Due to a poor understanding of the pathogenesis of $\mathrm{ONFH}$, a definite and effective treatment protocol to manage the disease has not been formed. Therefore, it is critical to find a method to predict $\mathrm{ONFH}$ in femoral neck fracture patients.
For early-stage $\mathrm{ONFH}$, a satisfactory prognosis can be achieved if the appropriate treatment is employed. Although, there have been several methods to predict fracture-induced $\mathrm{ONFH}$, they are inaccurate, invasive, complicated and narrowly used (Watanabe et al., 2007; Ehlinger et al., 2011).

It has been proven that regional osteogenesis and vasculogenesis can occur and can be detected in peripheral circulation in vivo as well. The systemic changes of these osteogenic and vasculogenic factors are significant for reflecting intrinsic functions. Among proangiogenic growth factors, Vascular Endothelial Growth Factor (VEGF) has emerged as a prominent mediator of angiogenesis and a potential therapeutic tool to enhance neovascularization. VEGF which can induce endothelial cell proliferation and capillary permeability could also influence the progress of fracture healing via multiple pathways. Suzuki et al. (2004) have shown that VEGF can promote surgical angiogenesis in necrotic bone. Alternatively, Katsube et al. (2005) employed VEGF gene transfer to enhance surgical revascularization of necrotic bone. BMP-2 is structurally related to the TGF $\beta$

Corresponding Author: C.Q. Zhang, Department of Orthopedic Surgery, Shanghai Sixth People's Hospital, Jiao Tong University, No. 600 Yishan Road, 200233 Shanghai, China 
superfamily and plays a critical role in osteoinductive activity and morphogenesis (Linkhart et al., 1996). In vivo, BMP-2 is secreted by cells from developing bone and is elevated at fracture sites in skeletally mature individuals, however it is down-regulated in nonunion patients (Kwong et al., 2009). Additionally, the systemic expression of BMP-2 is ill-defined in fracture-induced ONFH. The Runx (runt-related protein) family of transcription factors plays important roles in various tissues and cell lineages. Runx-2 is essential for the commitment of multipotent mesenchymal cells into the osteoblastic lineage and inhibits adipocyte differentiation. Runx-2 induces the gene expression of bone matrix proteins and maintains the osteoblastic cells in an immature stage (Komori, 2006). Runx1-expressing cells were detected in the periosteal callus together with Runx-2 expression and it was concluded that Runx1-expressing precursors first appear in the peristeal callus as an early fracture repair response (Shintaku et al., 2011).

However, the manner of local changes of Runx-2 is unclear in fracture-induced ONFH. Previous studies have also indicated that IGF-1 could stimulate osteoblastic cell proliferation and differentiated functions such as type I collagen expression (Linkhart et al., 1996). Meanwhile, IGF-1 may also be important for mediating systemic hormones on bone formation (Canalis et al., 1991).

In this prospective study, systemic and regional responses of osteogenic and angiogenic factors were detected in a canine model of femoral neck fractures which were treated at different fracture-to-surgery intervals. The occurrence of fracture-induced ONFH was observed after interval fixation at different injury-to-surgery intervals. Meanwhile, the relationship between traumatic $\mathrm{ONFH}$ and systemic changes in VEGF and IGF-1 as well as the local expression of BMP- 2 and Runx-2 were explored. Predictors for fracture-induced ONFH were discussed as well. This research protocol was approved by the ethics committee of our hospital.

\section{MATERIALS AND METHODS}

Animals: Beagles from the Laboratory Animal Center of the hospital were used as the Canine Model and had an average age of 2.5 years and body weight of $15.5 \mathrm{~kg}$ (range, 14-17 kg). All animals had no special history of diseases and were housed separately. Before the day of each operation, the dogs were fasted and penicillin sodium was injected intramuscularly. Twenty four dogs were divided randomly and equally into three groups for the following operational management and another two animals were used as control animals.
Operational methods and sample acquisition: Under aseptic conditions, a $6 \mathrm{~cm}$ long L-shape skin incision was made centered to the left of the great trochanter to expose the hip joint. The tensor fascia lata was split along the length of its bundles and the gluteus medius muscle was detached partially from the great trochanter. The hip joint capsule was opened and electrocoagulation of soft tissue attachments at the base of the femoral neck was performed circumferentially to interrupt the extraosseous blood supply to the femoral head, however the medial and lateral circumflex arteries were not revealed and the ligamentum teres was kept intact. A low-speed drill was employed to fracture the femoral neck at the narrow base. The fractures were reduced and stabilized by three parallel Kirschner pins immediately in the eight dogs in group A. An early operation was performed 3 days later in group B and 3 weeks later in group $C$ which was defined as a late operation. The femoral neck fractures were left untreated in the two control dogs. The maneuvers were directly visible for assurance of anatomical reduction and reliable stabilization. All operations were completed by one team of surgeons. Postoperatively, all animals were housed separately and injected with penicillin sodium for prophylactic control of infection. In each group, the femoral head from two dogs was harvested after it was fractured immediately (normal femoral head, group A), after 3 days (group B) and after 3 weeks (group C).

Venous blood samples from all dogs were collected via the peripheral venous preoperatively and on postoperative day $1,2,3,7,14$ and 21. Samples were drawn between 8 and 9 a.m., after an overnight fast. Serum was separated from other blood components by centrifugation at $1500 \mathrm{rpm}$ for $10 \mathrm{~min}$, aliquoted and stored at $-80^{\circ} \mathrm{C}$.

Measurement of serum concentrations of VEGF and IGF-1: Serum levels of VEGF and IGF-1 were quantified using commercially available ELISA kits (Xitang, Shanghai). Assays were performed according to the manufacturer's instructions and suggested serum dilutions. All samples were measured in triplicate.

Radiological examination: All animals underwent radiological examination 2 weeks postoperatively and then monthly for 3 times. Radiologically, the sclerotic rim is a reactive bone remodeling at the necrotic-viable osseous junction. This pattern characterizes the stage II according to the modified Ficat-Arlet, Steinberg's and ARCO systems. The presence of the crescent sign in the absence of segmental flattening, classifies the lesion as stage III in 
all major staging systems. For more advanced cases, the femoral head would collapse and deform with joint space narrowing.

Histological examinations: After the last radiological examination, all animals were euthanized and the bilateral femoral heads were harvested for histological examination. For microscopic examination, tissue samples were obtained from the zone of weight-bearing and the center of the femoral head. The samples were fixed with $10 \%$ formalin for 1 week and decalcified with $5 \mu \mathrm{M}$ EDTA solution for 4 weeks. The specimens were embedded in paraffin, cut into $4 \mu \mathrm{m}$ sections and stained with Hematoxylin and Eosin (HE).

Immunohistochemistry of BMP-2 and Runx2: For immunohistochemistry, each section was de-waxed, irradiated at $750 \mathrm{~W}$ in a microwave oven with $3 \%$ hydrogen peroxide in $0.01 \mathrm{M}$ sodium citrate buffer ( $\mathrm{pH}$ 6.0) for $5 \mathrm{~min}$ and immunostained with a monoclonal anti-BMP-2/Runx-2 antibody (Hope, Zhenjiang, China) to detect osteogenesis in the femoral head.

Statistical analysis: All numerical data were presented as mean \pm Standard Deviation (SD). Student t-test was employed to compare parametric differences in different groups using SPSS 17.0 software (Chicago, IL).

\section{RESULTS AND DISCUSSION}

General: No animals experienced postoperative complications and all fractures came to union by the last radiological examination. Radiologically, no animals showed $\mathrm{ONFH}$ in the treated groups, however both untreated animals had characteristic osteonecrosis.

Systemic expression of VEGF and IGF-1: After femoral neck fractures, the serum concentration of VEGF was down-regulated which was statistically different than pre-fracture levels $\left(157.57 \pm 22\right.$. $\left.40 \mathrm{pg} \mathrm{mL}^{-1}\right)$. In group A, VEGF maintained a lower level and gradually rose to $110.75 \pm 19.15 \mathrm{pg} \mathrm{mL}^{-1}$ on day 21 which was also statistically lower than that at the pre-fracture level. In group B, VEGF expression decreased after the femoral neck fractured but gradually climbed to $124.27 \pm 15.82 \mathrm{pg} \mathrm{mL}^{-1}$ on day 21 . For animals in group $\mathrm{C}$, the response manner of VEGF got similarity to that in group B before the fractures were but VEGF expression decreased slightly postoperatively and then climbed higher (Fig. 1a). Unlike VEGF, IGF-1 expression in group
A remained at a low level until the 7 th day after the femoral neck fractures. Then, the level of IGF-1 rose to $130.73 \pm 18.08 \mathrm{ng} \mathrm{mL}^{-1}$ on day 21 which was statistically lower than the pre-fracture concentration (198.21 $\pm 21.33 \mathrm{ng} \mathrm{mL} \mathrm{mL}^{-1}$ ). Similarly, in group B, IGF-1 decreased before the reduction and internal fixation of the fractures, continued to decrease postoperatively and then rose slightly. In group $\mathrm{C}$, the expression of IGF-1 shared great similarity and dropped slightly after operative management (Fig. 1b).

For untreated animals, there were no statistical differences concerning VEGF and IGF-1 expression when compared to those in groups A-C ( $\mathrm{p}>0.05$, respectively).

Histological examination: HE staining indicated that there was one animal in each treated group that developed osteonecrosis with characteristics of an accumulation of bone marrow cell debris, empty lacunae and/or ghost nuclei in the lacunae and an increase in the fat cells of the bone marrow. Morphologically, the surface of the cartilage in groups A-C remained intact, however it lost continuity for untreated animals, both of which developed osteonecrosis (Fig. 2).

For immunohistochemical staining of BMP-2 and Runx-2, ten fields of positive cells were randomly counted by two researchers and the total value was used for statistical analysis. Local expression of BMP-2 and Runx-2 was not statistically significant in groups $\mathrm{A}, \mathrm{B}$ and $\mathrm{C}$. Moreover, there were no statistical differences in BMP-2 and Runx-2 positive cells in untreated animals when compared to treated animals (Fig. 3).

Traumatic osteonecrosis of the femoral head is the main cause of poor prognosis following femoral neck fractures (Bachiller et al., 2002). Although, surgical technique has greatly advanced in past decades, the incidence of fracture-induced $\mathrm{ONFH}$ has not been significantly reduced. Due to the unclear pathogenesis of the disease, a definite and effective treatment protocol has not been formed, especially for advanced-stage $\mathrm{ONFH}$. Degeneration of the hip joint can trigger ongoing pain and restricted motion which usually requires artificial joint replacement in young, active patients. Therefore, a simple, accurate and non-invasive method is needed to predict fracture-induced $\mathrm{ONFH}$. Although, several invasive methods to predict $\mathrm{ONFH}$ following femoral neck fractures have been reported (Ehlinger et al., 2011), there have been no clinical results based on these methods.

The pathogenesis of ONFH is still unknown, however for fracture-induced $\mathrm{ONFH}$, the destruction of the blood 

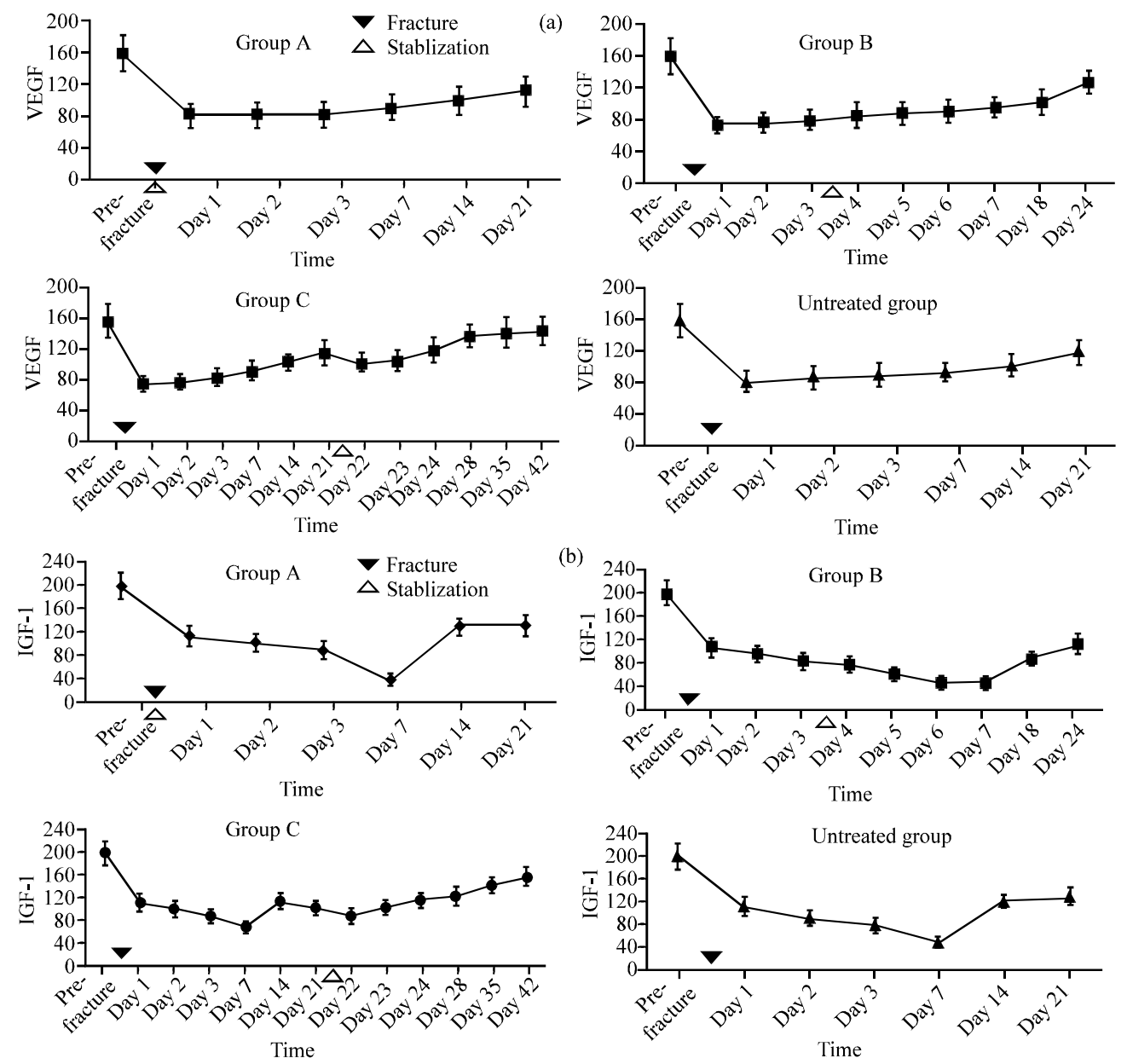

Fig. 1: Systemic response of VEGF and IGF-1 detected by ELISA; a) VEGF expression (pg mL ${ }^{-1}$ ); b) IGF-1 expression (ng $\mathrm{mL}^{-1}$ )

supply causes serum deprivation and hypoxia that might lead to necrosis and apoptosis of osteocytes. Therefore, the reactions of vessels and osteocytes could be expressed systemically and locally. Weiss et al. (2009) researched the systemic angiogenic response during bone healing and indicated that $\mathrm{bFGF}$ and PDGF-AB were significant predictors of fracture nonunion while VEGF, ANG, Ang-2 and PTN were not significant. Researchers investigated the angiogenic factor VEGF during femoral neck fractures and fixation and detected systemic IGF-1 and local BMP-2 and Runx-2, indicative of osteogenesis. Systematic changes in VEGF and IGF-1 expression were considerably similar following femoral neck fractures; they were both down-regulated in the short term and the intervention of fracture reduction and fixation did not affect their expression. It is known that VEGF is the principal stimulator of angiogenesis (Street et al., 2002; Radke et al., 2006; Keramaris et al., 2008) which is adversely regulated by fractures. Although, it has been shown that angiogenesis is a basic component for fracture union and the prevention of ONFH which could be promoted via the local release of VEGF (Kempen et al., 2009), there are few studies that report the systemic response of VEGF expression continuously and prospectively. It could be concluded that additional VEGF treatment is beneficial for fracture union and the prevention of ONFH. IGF-1 stimulates preosteoblastic cell replication and increases the cell population capable of synthesizing bone matrix (Linkhart et al., 1996). Recent studies have indicated that IGF-1 could be widely employed in clinical and functional evaluations (Kanazawa et al., 2011; Di Monaco et al., 2009). Meanwhile, low serum IGF-1 was associated with an increased risk of incident fractures (Ohlsson et al., 2011). Therefore, based on these results, additional IGF-1 treatment for the prevention of fractures and fracture 


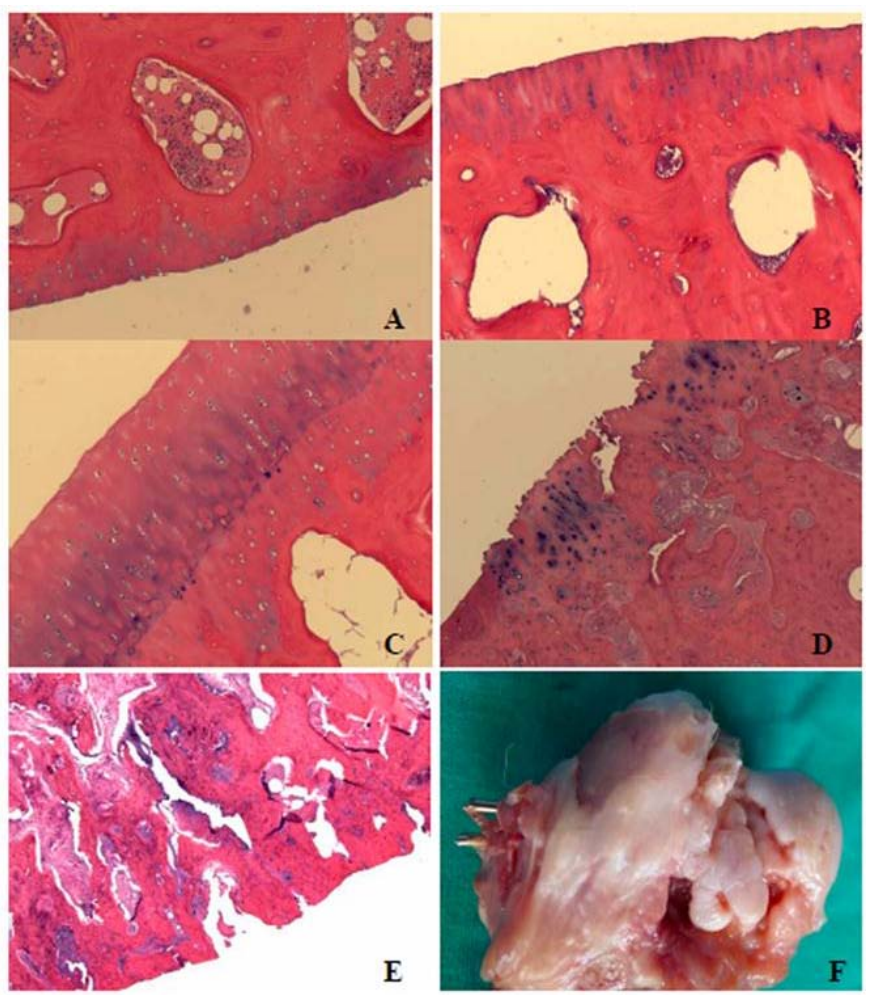

Fig. 2: HE staining and morphology of the femoral head. A-C) The femoral heads from group A-C were normal when fractures fused. However, for untreated animals; D) Discontinuity of cartilage; E) Microfracture of the trabeculae were detected; F) The femoral head maintained its smooth surface with cartilage formation at the fracture site

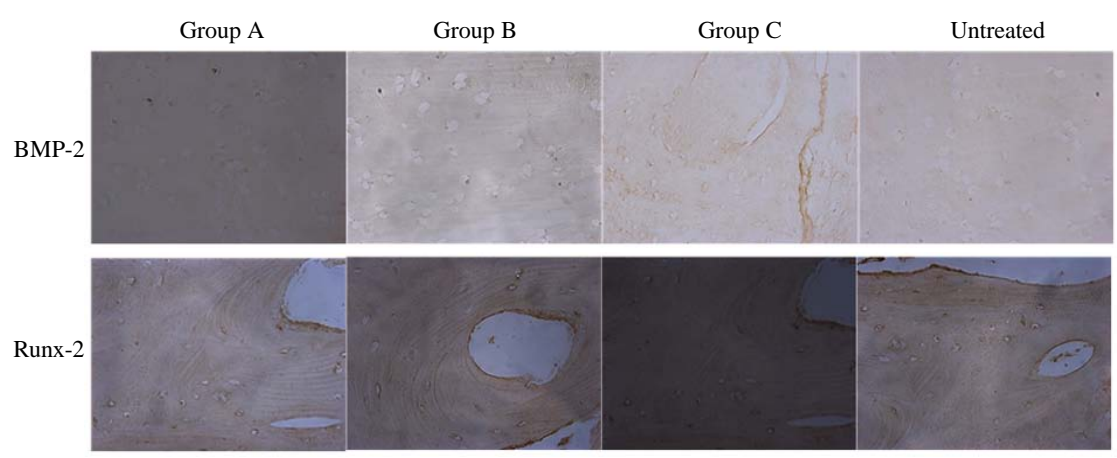

Fig. 3: Local expression of BMP-2 and Runx-2 by immunohistochemical staining. The numbers of BMP-2 and Runx-2-positive cells were similar in treated and untreated animals

induced complications is critical although, the course of the IGF-1 response was not affected by different injury-to-surgery intervals. Thus, VEGF and IGF-1 are useless as predictors of fracture-induced $\mathrm{ONFH}$. After femoral neck fractures were made, the femoral head was left in situ for further reduction and fixation. The head was harvested for histological examination in two animals from each group at the respective intervals. This present data indicated similar levels of local Runx-2 and BMP-2 expression. Interestingly, the effect of serum deprivation and hypoxia on osteogenesis is not significant. It is controversial that hypoxia might play a positive or negative effect on osteogenesis of precursor cells (Grayson et al., 2007; Holzwarth et al., 2010). Previously, a significant reduction in BMP-2 and BMP-1 4 expression in cartilaginous areas of nonhealing fractures compared to 
healing fractures was reported (Kwong et al., 2009). In this study when all fractures united, there was no difference in the expression of BMP-2 and Runx-2 and a newly restored balance had formed during the fracture union. Due to the similar characteristics of BMP-2 and Runx-2 expression, it is useless to employ them as predictors of fracture-induced $\mathrm{ONFH}$.

\section{CONCLUSION}

The relationship between systemic and local angiogenesis and osteogenesis following femoral neck fractures is unclear and their mechanisms on fractureinduced ONFH might be more complicated. This is the first prospective study to explore the relationship between the injury-to-surgery interval and its effect on induced $\mathrm{ONFH}$ as well as the possible underlying mechanism of systemic and regional angiogenesis/osteogenesis differences following femoral neck fractures. The systemic expression of VEGF and IGF-1 had similar characteristics when fractures were either treated at different intervals or left untreated. Meanwhile, the regional expressions of BMP-2 and Runx-2 were also similar when fractures were repaired. These data suggest that systemic and regional angiogenesis/osteogenesis respond similarly to different injury-to-surgery intervals. Therefore, researchers cautiously conclude that angiogenic and osteogenic responses might not be responsible for fracture induced $\mathrm{ONFH}$ and they cannot be employed as a predictor of traumatic $\mathrm{ONFH}$.

However, anatomical and biomechanical differences between the hips of canines and human beings limit the clinical significance of this report and the number of detected factors and animals was relatively small. Therefore, a prospective and randomized controlled study using a larger number of animals and factors should be performed in the future to determine the intrinsic links between angiogenic and osteogenic responses and induced $\mathrm{ONFH}$ and the predicted value of continuous monitoring of these factors.

\section{ACKNOWLEDGEMENT}

The current research was financially supported by a Creative Research Scholarship for Ph.D. Candidates of Shanghai Jiao Tong University School of Medicine (No.: BXJ2011038).

\section{REFERENCES}

Bachiller, F.G., A.P. Caballer and L.F. Portal, 2002. Avascular necrosis of the femoral head after femoral neck fracture. Clin. Orthop. Relat. Res., 399: 87-109.
Canalis, E., T.L. McCarthy and M. Centrella, 1991. Growth factors and cytokines in bone cell metabolism. Annu. Rev. Med., 42: 17-24.

Di Monaco, M., F. Vallero, R. Di Monaco, R. Tappero and A. Cavanna, 2009. Serum levels of insulin-like growth factor-I are positively associated with functional outcome after hip fracture in elderly women. Am. J. Phys. Med. Rehabil., 88: 119-125.

Ehlinger, M., T. Moser, P. Adam, G. Bierry, A. Gangi, M. de Mathelin and F. Bonnomet, 2011. Early prediction of femoral neck avascular necrosis following neck fracture. Orthop. Traumatol. Surg. Res., 97: 79-88.

Grays on, W.L., F. Zhao, B. Bunnell and T. Ma, 2007. Hypoxia enhances proliferation and tissue formation of human mesenchymal stem cells. Biochem. Biophys. Res. Commun., 358: 948-953.

Holzwarth, C., M. Vaegler, F. Gieseke, S.M. Pfister, R. Handgretinger, G. Kerst and I. Muller, 2010. Low physiologic oxygen tensions reduce proliferation and differentiation of human multipotent mesenchymal stromal cells. BMC Cell Biol., Vol. 11. 10.1186/14712121-11-11

Jones, L.C. and D.S. Hungerford, 2007. The pathogenesis of osteonecrosis. Instr. Course Lect., 56: 179-196.

Kanazawa, I., T. Yamaguchi and T. Sugimoto, 2011. Serum insulin-like growth factor-I is a marker for assessing the severity of vertebral fractures in postmenopausal women with type- 2 diabetes mellitus. Osteoporos. Int., 22: 1191-1198.

Katsube, K., A.T. Bishop, R.D. Simari, S. Yla-Herttuala and P.F. Friedrich, 2005. Vascular Endothelial Growth Factor (VEGF) gene transfer enhances surgical revascularization of necrotic bone. J. Orthop. Res., 23: 469-474.

Kempen, D.H.R., L. Lu, A. Heijink, T.E. Hefferan and L.B. Creemers et al., 2009. Effect of local sequential VEGF and BMP-2 delivery on ectopic and orthotopic bone regeneration. Biomaterials, 30: 2816-2825.

Keramaris, N.C., G.M. Calori, V.S. Nikolaou, E.H. Schemitsch and P.V. Giannoudis, 2008. Fracture vascularity and bone healing: A systematic review of the role of VEGF. Injury, 39: S45-S57.

Komori, T., 2006. Regulation of osteoblast differentiation by transcriptional factors. J. Cell. Biochem., 99: 1233-1239.

Kwong, F.N., J.A. Hoyland, A.J. Freemont and C.H. Evans, 2009. Altered relative expression of BMPs and BMP inhibitors in cartilaginous areas of human fractures progressing towards nonunion. J. Orthop. Res., 27: 752-757. 
Kyle, R.F., 2009. Fractures of the femoral neck. Instr. Course Lect., 58: 61-68.

Linkhart, T.A., S. Mohan and D.J. Baylink, 1996. Growth factors for bone growth and repair: IGF, TGF-beta and BMP. Bone, 19: 1S-12S.

Lu-Yao, G.L., R.B. Keller, B. Littenberg and J.E. Wennberg, 2004. Outcomes after displaced fractures of the femoral neck. A meta-analysis of one hundred and six published reports. J. Bone Joint Surg. Am., 76: $15-25$.

Ohlsson, C., D. Mellstrom, D. Carlzon, E. Orwoll, O. Ljunggren, M.K. Karlsson and L. Vandenput, 2011. Older men with low IGF-1 have an increased risk of incident fractures: The MrOS Sweden study. J. Bone Miner. Res., 26: 865-872.

Radke, S., A. Battmann, S. Jatzke, J. Eulert, F. Jakob and N. Schutze, 2006. Expression of the angiomatrix and angiogenic proteins CYR61, CTGF and VEGF in osteonecrosis of the femoral head. J. Orthop. Res., 24: $945-952$.

Shintaku, Y., T. Murakami, T. Yanagita, N. Kawanabe and T. Fukunaga et al., 2011. Sox9 expression during fracture repair. Cells Tissues Organs, 194: 38-48.
Street, J., M. Bao, L. de Guzman, S. Bunting and J.F.V. Peale et al., 2002. Vascular endothelial growth factor stimulates bone repair by promoting angiogenesis and bone turnover. Proc. Natl. Acad. Sci., 99: 9656-9661.

Suzuki, O., A.T. Bishop, T. Sunagawa, K. Katsube and P.F. Friedrich, 2004. VEGF-promoted surgical angiogenesis in necrotic bone. Microsurgery, 24: 85-91.

Upadhyay, A., P. Jain, P. Mishra, L. Maini, V.K. Gautum and B.K. Dhaon, 2004. Delayed internal fixation of fractures of the neck of the femur in young adults. A prospective, randomized study comparing closed and open reduction. J. Bone Joint Surg. Br., 86: 1035-1040.

Watanabe, Y., Y. Terashima, N. Takenaka, M. Kobayashi and T. Matsushita, 2007. Prediction of avascular necrosis of the femoral head by measuring intramedullary oxygen tension after femoral neck fracture. J. Orthop. Trauma., 21: 456-461.

Weiss, S., G. Zimmermann, T. Pufe, D. Varoga and P. Henle, 2009. The systemic angiogenic response during bone healing. Arch. Orthop. Trauma Surg., 129: 989-997. 